\title{
New Bounds for the Resolvent Energy of Graphs
}

\author{
E. H. Zogić, E. R. Glogić
}

\begin{abstract}
The resolvent energy of a graph $G$ of order $n$ is defined as $E R(G)=\sum_{i=1}^{n}\left(n-\lambda_{i}\right)^{-1}$, where $\lambda_{1} \geq \lambda_{2} \geq \cdots \geq \lambda_{n}$ are the eigenvalues of $G$. Lower and upper bounds for the resolvent energy of a graph, which depend on some of the parameters $n, \lambda_{1}, \lambda_{n}, \operatorname{det}\left(\mathscr{R}_{A}(n)\right)=\prod_{i=1}^{n} \frac{1}{n-\lambda_{i}}$, are obtained.
\end{abstract}

Keywords: resolvent energy, graph, inequalities

\section{Introduction}

Let $M$ be a square matrix of order $n$. The resolvent matrix, $\mathscr{R}_{M}(z)$, of matrix $M$ is defined as [9]

$$
\mathscr{R}_{M}(z)=\left(z I_{n}-M\right)^{-1},
$$

where $I_{n}$ is the unit matrix of order $n$ and $z$ a complex variable. As easily seen, $\mathscr{R}_{M}(z)$ is also a matrix of order $n$, that exists for all values of $z$ except when $z$ coincides with an eigenvalue of $M$.

Let $G$ be the simple graph, $A$ its adjacency matrix and $\lambda_{1} \geq \lambda_{2} \geq \cdots \geq \lambda_{n}$ eigenvalues of $A$. The resolvent matrix, $\mathscr{R}_{A}(z)$, is defined as

$$
\mathscr{R}_{A}(z)=\left(z I_{n}-A\right)^{-1},
$$

and its eigenvalues are

$$
\frac{1}{z-\lambda_{i}}, i=1,2, \ldots, n
$$

Bearing in mind that $\lambda_{i} \leq n-1$ for all $i=1,2, \ldots, n$, [4], we could choose $z=n$. Now we have that $\frac{1}{n-\lambda_{i}}, i=1,2, \ldots, n$ are the eigenvalues of matrix $\mathscr{R}_{A}(n)=\left(n I_{n}-A\right)^{-1}$ and $\operatorname{det}\left(\mathscr{R}_{A}(n)\right)=\prod_{i=1}^{n} \frac{1}{n-\lambda_{i}}$.

\footnotetext{
Manuscript received June 17, 2017. ; accepted October 21, 2017.

E. H. Zogić and E. R. Glogić are with the State University of Novi Pazar, Department of Mathematical Sciences, Novi Pazar, Serbia
} 
Let $G$ be a graph on $n$ vertices, $n>1$, with eigenvalues $\lambda_{1}, \lambda_{2}, \ldots, \lambda_{n}$. Its resolvent energy is [7]

$$
E R(G)=\sum_{i=1}^{n} \frac{1}{n-\lambda_{i}}
$$

Some remarkable properties of $E R(G)$ were revealed in [7]. There are results about defining $E R(G)$ via spectral moments and characteristic polynomial of graphs, and some bounds for the $E R(G)$ in terms of parameters $n, m, n_{0}$, where $m$ is the number of edges and $n_{0}$ is a nullity of the graph. Additional properties of $E R(G)$ can be also found in the recent papers $[1,5,6,11]$.

In this paper, we obtained some new lower and upper bounds for the resolvent energy of a graph in terms of $n, \lambda_{1}, \lambda_{n}$ and $\operatorname{det}\left(\mathscr{R}_{A}(n)\right)$.

\section{Some common inequalities and preliminary lemmas}

Now, we introduce some common inequalities which we need for our proofs in the section of main results.

Lemma 2.1 [8] Let $a_{i}, r, R \in \mathbb{R}, 0<r \leq a_{i} \leq R, i=1, \ldots, n$. Then

$$
n \sum_{i=1}^{n} a_{i}^{2}-\left(\sum_{i=1}^{n} a_{i}\right)^{2} \geq \frac{n}{2}(R-r)^{2}
$$

Lemma 2.2 [8] Let $a_{i}, p_{i}, r, R \in \mathbb{R}, 0<r \leq a_{i} \leq R, i=1, \ldots, n, \sum_{i=1}^{n} p_{i}=1$. Then

$$
\sum_{i=1}^{n} p_{i} a_{i}+r R \sum_{i=1}^{n} \frac{p_{i}}{a_{i}} \leq r+R
$$

Lemma 2.3 [10] Let $a_{i} \in \mathbb{R}^{+}, i=1, \ldots, n$. Then

$$
(n-1) \sum_{i=1}^{n} a_{i}+n\left(\prod_{i=1}^{n} a_{i}\right)^{\frac{1}{n}} \geq\left(\sum_{i=1}^{n} \sqrt{a_{i}}\right)^{2} \geq \sum_{i=1}^{n} a_{i}+n(n-1)\left(\prod_{i=1}^{n} a_{i}\right)^{\frac{1}{n}} .
$$

Lemma 2.4 [8] Let $a_{i}, p_{i}, r, R \in \mathbb{R}, 0<r \leq a_{i} \leq R, i=1, \ldots, n$, $\sum_{i=1}^{n} p_{i}=1$. Then

$$
\sum_{i=1}^{n} p_{i} a_{i} \sum_{i=1}^{n} \frac{p_{i}}{a_{i}} \leq \frac{1}{4}\left(\sqrt{\frac{R}{r}}+\sqrt{\frac{r}{R}}\right)^{2}
$$

Lemma 2.5 [3] Let $0<a_{1} \leq \cdots \leq a_{i} \leq \cdots \leq a_{k} \leq \cdots \leq a_{n}, p_{1}, p_{2}, \ldots, p_{n}$ be positive real numbers such that $p_{1}+p_{2}+\cdots+p_{n}=1$ and $Q_{i}=p_{1}+p_{2}+\cdots+p_{i}, R_{k}=p_{k}+p_{k+1}+\cdots p_{n}$. Then

$$
\frac{p_{1}}{a_{1}}+\frac{p_{2}}{a_{2}}+\cdots+\frac{p_{n}}{a_{n}}-\frac{1}{p_{1} a_{1}+p_{2} a_{2}+\cdots+p_{n} a_{n}} \geq \frac{Q_{i} R_{k}\left(a_{k}-a_{i}\right)^{2}}{a_{i} a_{k}\left(Q_{i} a_{i}+R_{k} a_{k}\right)},
$$


with equality for $a_{1}=a_{2}=\cdots=a_{i}, a_{k}=a_{k+1}=\cdots=a_{n}, a_{i+1}=a_{i+2}=\cdots=a_{k-1}=$ $\frac{Q_{i} a_{i}+R_{k} a_{k}}{Q_{i}+R_{k}}$.

Lemma 2.6 [2] Let $p_{1}, p_{2}, \ldots, p_{n}$ be non-negative real numbers and $a_{1}, a_{2}, \ldots, a_{n}$ and $b_{1}, b_{2}, \ldots, b_{n}$ real numbers with the properties $0<r_{1} \leq a_{i} \leq R_{1}<+\infty$ and $0<r_{2} \leq b_{i} \leq R_{2}<+\infty$ for each $i=1,2, \ldots, n$. Further, let $S$ be a subset of $I_{n}=\{1,2, \ldots, n\}$ which minimizes the expression $\left|\sum_{i \in S} p_{i}-\frac{1}{2} \sum_{i=1}^{n} p_{i}\right|$. Then

$$
\left|\sum_{i=1}^{n} p_{i} \sum_{i=1}^{n} p_{i} a_{i} b_{i}-\sum_{i=1}^{n} p_{i} a_{i} \sum_{i=1}^{n} p_{i} b_{i}\right| \leq\left(R_{1}-r_{1}\right)\left(R_{2}-r_{2}\right) \sum_{i \in S} p_{i}\left(\sum_{i=1}^{n} p_{i}-\sum_{i \in S} p_{i}\right) .
$$

Lemma 2.7 [4] A graph has one eigenvalue if and only if it is totally disconnected. A graph has two distinct eigenvalues $\lambda_{1}>\lambda_{2}$ with multiplicities $m_{1}$ and $m_{2}$ if and only if it consists of $m_{1}$ complete graphs of order $\lambda_{1}+1$. In that case, $\lambda_{2}=-1$ and $m_{2}=m_{1} \lambda_{1}$.

Lemma 2.8 [8] Let $a=\left(a_{i}\right), b=\left(b_{i}\right), c=\left(c_{i}\right)$ be three sequences of real numbers of the same monotonicity and $p=\left(p_{i}\right)$ sequence of real number. Then

$$
\left(\sum_{i=1}^{n} p_{i}\right)^{2} \sum_{i=1}^{n} p_{i} a_{i} b_{i} c_{i} \geq \sum_{i=1}^{n} p_{i} a_{i} \sum_{i=1}^{n} p_{i} b_{i} \sum_{i=1}^{n} p_{i} c_{i}
$$

If $a=\left(a_{i}\right)$ and $b=\left(b_{i}\right)$ are oppositely ordered, then the sense of ineqality (7) reverses.

\section{Main results}

We represent some new lower and upper bounds for the resolvent energy of graphs.

Theorem 3.1 Let $G$ be a graph on $n$ vertices with eigenvalues $\lambda_{1} \geq \lambda_{2} \geq \cdots \geq \lambda_{n}$. Then

$$
E R(G) \geq n\left(\operatorname{det}\left(\mathscr{R}_{A}(n)\right)\right)^{\frac{1}{n}}+\frac{n}{2(n-1)} \cdot \frac{\left(\sqrt{n-\lambda_{n}}-\sqrt{n-\lambda_{1}}\right)^{2}}{\left(n-\lambda_{1}\right)\left(n-\lambda_{n}\right)}
$$

Equality is attained if and only if $G=\bar{K}_{n}$.

Proof. Let's consider inequalities (1) and (3), where we could choose $r=\frac{1}{\sqrt{n-\lambda_{n}}}, R=$ $\frac{1}{\sqrt{n-\lambda_{1}}}, a_{i}=\frac{1}{\sqrt{n-\lambda_{i}}}, i=1, \ldots, n$, to obtain

$$
\begin{gathered}
n \sum_{i=1}^{n} \frac{1}{n-\lambda_{i}}-\left(\sum_{i=1}^{n} \frac{1}{\sqrt{n-\lambda_{i}}}\right)^{2} \geq \frac{n}{2}\left(\frac{1}{\sqrt{n-\lambda_{1}}}-\frac{1}{\sqrt{n-\lambda_{n}}}\right)^{2} . \\
\left(\sum_{i=1}^{n} \frac{1}{\sqrt{n-\lambda_{i}}}\right)^{2} \geq \sum_{i=1}^{n} \frac{1}{n-\lambda_{i}}+n(n-1)\left(\prod_{i=1}^{n} \frac{1}{\sqrt{n-\lambda_{i}}}\right)^{\frac{1}{n}} .
\end{gathered}
$$


From the definition of the resolvent energy of graph, $E R(G)=\sum_{i=1}^{n} \frac{1}{n-\lambda_{i}}$, and by (9), we have

$$
\begin{aligned}
n E R & \geq\left(\sum_{i=1}^{n} \frac{1}{\sqrt{n-\lambda_{i}}}\right)^{2}+\frac{n}{2}\left(\frac{1}{\sqrt{n-\lambda_{1}}}-\frac{1}{\sqrt{n-\lambda_{n}}}\right)^{2} \\
& \geq E R+n(n-1)\left(\prod_{i=1}^{n} \frac{1}{n-\lambda_{i}}\right)^{\frac{1}{n}}+\frac{n}{2} \cdot \frac{\left(\sqrt{n-\lambda_{n}}-\sqrt{n-\lambda_{1}}\right)^{2}}{\left(n-\lambda_{1}\right)\left(n-\lambda_{n}\right)} \\
& =E R+n(n-1)\left(\operatorname{det}\left(\mathscr{R}_{A}(n)\right)\right)^{\frac{1}{n}}+\frac{n}{2} \cdot \frac{\left(\sqrt{n-\lambda_{n}}-\sqrt{n-\lambda_{1}}\right)^{2}}{\left(n-\lambda_{1}\right)\left(n-\lambda_{n}\right)}
\end{aligned}
$$

where in the second inequality we used (10). Now, it follows that

$$
E R(G) \geq n\left(\operatorname{det}\left(\mathscr{R}_{A}(n)\right)\right)^{\frac{1}{n}}+\frac{n}{2(n-1)} \cdot \frac{\left(\sqrt{n-\lambda_{n}}-\sqrt{n-\lambda_{1}}\right)^{2}}{\left(n-\lambda_{1}\right)\left(n-\lambda_{n}\right)} .
$$

If $G=\bar{K}_{n}$ then $E R\left(\bar{K}_{n}\right)=1$ and in (8) equality holds.

If equality holds in (8) then equality is attained in (9) i (10), from wich follows that $\lambda_{1}=\lambda_{2}=\cdots=\lambda_{n}$. By the Lemma 2.7 it follows that $G=\bar{K}_{n}$.

Theorem 3.2 Let $G$ be a graph on $n$ vertices with eigenvalues $\lambda_{1} \geq \lambda_{2} \geq \cdots \geq \lambda_{n}$. Then

$$
E R(G) \geq \frac{n^{2}+\left(n-\lambda_{1}\right)\left(n-\lambda_{n}\right)}{n\left(2 n-\lambda_{1}-\lambda_{n}\right)}
$$

Proof. Using Chebyshev inequality for 3 sequences (7) and using inequality (2), for $a_{i}=$ $\frac{1}{n-\lambda_{i}}, p_{i}=\frac{1}{n}, i=1, \ldots, n, r=\frac{1}{n-\lambda_{n}}, R=\frac{1}{n-\lambda_{1}}$ we obtain a lower bound (11).

Theorem 3.3 Let $G$ be a graph on $n$ vertices with eigenvalues $\lambda_{1} \geq \lambda_{2} \geq \cdots \geq \lambda_{n}$. Then

$$
E R(G) \geq 1+\frac{\left(\lambda_{n}-\lambda_{1}\right)^{2}}{\left(n-\lambda_{1}\right)\left(n-\lambda_{n}\right)\left(2 n-\lambda_{1}-\lambda_{n}\right)}
$$

Proof. For $a_{i}=n-\lambda_{i}, p_{i}=\frac{1}{n}, i=1, \ldots, n, Q_{i}=R_{k}=\frac{1}{n}$, the inequality (5) transforms into $E R(G) \geq 1+\frac{\left(\lambda_{n}-\lambda_{1}\right)^{2}}{\left(n-\lambda_{1}\right)\left(n-\lambda_{n}\right)\left(2 n-\lambda_{1}-\lambda_{n}\right)}$.

Theorem 3.4 Let $G$ be a graph on $n$ vertices with eigenvalues $\lambda_{1} \geq \lambda_{2} \geq \cdots \geq \lambda_{n}$. Then

$$
E R(G) \leq \frac{1}{4} \cdot \frac{\left(2 n-\lambda_{1}-\lambda_{n}\right)^{2}}{\left(n-\lambda_{1}\right)\left(n-\lambda_{n}\right)} .
$$

Proof. Using $p_{i}=\frac{1}{n}, a_{i}=\frac{1}{n-\lambda_{i}}, i=1, \ldots, n, r=\frac{1}{n-\lambda_{n}}, R=\frac{1}{n-\lambda_{1}}$, in the (4) we obtain the upper bound (13). 
Theorem 3.5 Let $G$ be a graph on $n$ vertices with eigenvalues $\lambda_{1} \geq \lambda_{2} \geq \cdots \geq \lambda_{n}$. Then

$$
\begin{gathered}
-\frac{\left(\lambda_{1}-\lambda_{n}\right)^{2}}{\left(n-\lambda_{1}\right)^{2}\left(n-\lambda_{n}\right)^{2}} \cdot \frac{n^{2}}{n-1} \alpha(n)+n\left(\operatorname{det}\left(\mathscr{R}_{A}(n)\right)^{\frac{1}{n}} \leq E R(G)\right. \\
\leq \frac{\left(\lambda_{1}-\lambda_{n}\right)^{2}}{\left(n-\lambda_{1}\right)^{2}\left(n-\lambda_{n}\right)^{2}} \cdot n^{2} \alpha(n)+n\left(\operatorname{det}\left(\mathscr{R}_{A}(n)\right)^{\frac{1}{n}} .\right.
\end{gathered}
$$

Proof. The proof follows from the inequality (6) for $a_{i}=b_{i}=\frac{1}{\sqrt{n-\lambda_{i}}}, i=1, \ldots, n, r_{1}=r_{2}=$ $\frac{1}{\sqrt{n-\lambda_{n}}}, R_{1}=R_{2}=\frac{1}{\sqrt{n-\lambda_{1}}}$.

\section{References}

[1] L. E. Allem, J. Capaverde, V. Trevisan, I. Gutman, E. Zogić, E. Glogić, Resolvent Energy of Unicyclic, Bicyclic and Tricyclic Graphs, MATCH Commun. Math. Comput. Chem. 77 (2017) 95-104.

[2] Andrica, D., Badea, C., Grüs inequality for positive linear functionals, Period. Math. Hungar, 19 (1988), no. 2, 155-167.

[3] V. Cirtoaje, The Best Lower Bound Depended on Two Fixed Variables for Jensens Inequality with Ordered Variables, Journal of Inequalities and Applications, 2010 (2010), 1-12.

[4] D. Cvetković, M. Doob, H. Sachs, Spectra of Graphs - Theory and Application, Academic Press, New York, 1980.

[5] Z. Du, Asymptotic Expressions for Resolvent Energies of Paths and Cycles, MATCH Commun. Math. Comput. Chem. 77 (2017) 85-94.

[6] M. Ghebleh, A. Kanso, D. Stevanović, On Trees with Smallest Resolvent Energy, MATCH Commun. Math. Comput. Chem. 77 (2017) 635-654.

[7] I. Gutman, B. Furtula, E. Zogić, E. Glogić Resolvent energy of graphs, MATCH Commun. Math. Comput. Chem. 75 (2016) 279-290.

[8] D. S. Mitrinović, P. Vasić, Analytic Inequalities, Springer, Berlin, 1970.

[9] T.S. Shores, Linear Algebra and Matrix Analysis, Springer, New York, 2007.

[10] B. Zhou, I. Gutman, T. Aleksić A note on Laplacian energy of graphs, MATCH Commun. Math. Comput. Chem. 60 (2008) 441-446.

[11] Z. Zhu, Some extremal properties of the resolvent energy, Estrada and resolvent Estrada indices of graphs, Journal of Mathematical Analysis and Applications 447 (2017) 957-970. 